\title{
A PESQUISA CIENTÍFICA SOBRE O MEIO RURAL E ASPECTOS LIGADOS À SUSTENTABILIDADE
}

\author{
Jorge André Thomas \\ Sandra Mara Stocker Lago** \\ Loreni Teresinha Brandalise ${ }^{* * *}$
}

\begin{abstract}
RESUMO: Historicamente a agricultura sofreu diversas modificações causadas por pressões políticas, sociais e econômicas, passando de uma atividade de moldes primitivos para uma atividade com intensa aplicação de tecnologia, fazendo o Brasil ocupar a sétima posição na economia mundial. Neste contexto, o objetivo deste estudo é analisar as produções científicas brasileiras sobre o meio rural e sobre aspectos da sustentabilidade, em que, para seu alcance, buscou-se identificar os estudos publicados entre 2010 e 2015 , no banco de teses e dissertações da Capes e, entre 2006 e 2015 nas revistas brasileiras com Qualis/Capes A1 e A2 na área de Administração, contendo as palavras "rural" e "sustentabilidade". O estudo enquadrase como exploratório, bibliográfico, quantitativo e qualitativo. Conclui-se que são poucos os estudos nesta área que se voltam para o meio rural. A maior parte dos estudos do banco de teses e dissertações da Capes concentrou-se na região Nordeste enquanto que, dos artigos, a maior parte ocorreu no Sul do Brasil. O baixo volume de estudos sugere a falta de interesse dos pesquisadores em realizar estudos, bem como das editoras e avaliadores das revistas em publicá-los. Sugere-se a continuidade de uma revisão bibliográfica neste tema, abrangendo outras bases de dados.
\end{abstract}

PALAVRAS-CHAVE: Análise Bibliográfica; Rural; Sustentabilidade.

\section{SCIENTIFIC RESEARCH ON THE RURAL SEGMENT AND ASPECTS LINKED TO SUSTAINABILITY}

\footnotetext{
ABSTRACT. Agriculture has endured several modifications caused by political, social and economic pressures. It passed from an activity with primitive characteristics towards an activity with intense technological applications. In fact, Brazil ranks

Mestre em Administração, modalidade profissional; Docente do curso de graduação em Ciências Contábeis na Faculdade Luterana Rui Barbosa (FALURB), Brasil.

** Doutora em Desenvolvimento Regional e Agronegócio; Docente permanente do Programa de Mestrado Profissional em Administração da Universidade Estadual do Oeste do Paraná (Unioeste), campus de Cascavel, Brasil. E-mail: smstocker@uol.com.br

*** Doutora em Engenharia de Produção; Docente permanente do Programa de Mestrado Profissional em Administração da Universidade Estadual do Oeste do Paraná (Unioeste), campus de Cascavel, Brasil.
} 
seventh in world economy. Current analysis investigates Brazil's scientific production on the rural segment and on certain aspects of sustainability. Studies published between 2010 and 2015 were searched in theses and dissertations in CAPES databases, and between 2006 and 2015 in Brazilian journals with Qualis/ Capes A1 and A2 in Administration, using keywords "rural" and "sustainability" in current exploratory, quantitative and qualitative research. Results show that studies on the theme are few. Most studies in theses and dissertation in the Capes database are concentrated within the Northeastern region of Brazil, whereas most articles are concentrated in southern Brazil. Low number of studies suggests lack of interest of researchers, publishing houses and publishers. Bibliographic reviews on the theme with other databases should be continued.

KEY WORDS: Bibliographic analysis; Rural area; Sustainability.

\section{INTRODUÇÃO}

A agricultura ao longo do tempo sofreu diversas modificações, pois suas distintas configurações resultaram em complexas transformações que envolveram a produção de alimentos, geração de emprego e renda, paisagem, particularidades sociais nas mais diferentes realidades agrárias existentes. Tais transformações_foram influenciadas por questões políticas, socioeconômicos e culturais, passando de uma agricultura de moldes primitivos para um modelo tecnológico, com o uso abusivo de insumos e aplicação de tecnologias (LIMA, 2011).

A importância da agricultura é tamanha no Brasil, que o leva à posição de sétima economia mundial, com um PIB de US\$ 2,4 trilhões, o que representa aproximadamente $50 \%$ da economia latino-americana. Adicionalmente, o país possui um amplo mercado consumidor, na casa de 201 milhões de cidadãos, estando localizado no Sul do continente sul americano, o que o favorece em termos de acesso aos mercados da América Latina e África (RENAI, 2014).

Neste contexto, destaca-se também a agricultura familiar que Roesler (2009) classifica como sendo um modelo de produção em que a família é, ao mesmo tempo, proprietária dos meios de produção e responsável pelo trabalho, gerando a produção de acordo com a estrutura disponível. Lamarche (1993) corrobora afirmando que, 
na agricultura familiar, a família é quem decide e age, bem como organiza e direciona os esforços para o alcance dos objetivos da unidade de produção.

A agricultura familiar foi considerada por muitos anos apenas uma agricultura de subsistência, porém, o segmento é hoje responsável por uma parcela significativa da produção agrícola e da geração de emprego no campo (CARVALHO; SANTOS; CARVALHO, 2015).

Para Santos, Marion e Segatti (1996), a administração também está associada à propriedade rural, uma vez que o papel de um administrador é planejar, controlar, decidir e avaliar os resultados das decisões. Mesmo nas pequenas propriedades rurais, onde a agricultura familiar predomina, por mais simples que sejam as boas práticas de gestão são muito importantes (LAMARCHE, 1993).

Ao tratar-se de práticas sustentáveis, na agricultura familiar ou nas pequenas propriedades rurais é que estas se iniciam, a partir das ações locais, em que as práticas sustentáveis somadas influenciam o contexto global, na medida em que o indivíduo se enxerga como parte de um processo mais amplo, no qual, as suas ações, por menores que sejam, são responsáveis pela manutenção e alterações na qualidade de vida das gerações futuras (KUMMER, 2007).

Estas ações, embora que minoritárias e ainda incipientes em alguns contextos da produção agrícola brasileira, têm evoluído e sendo reconhecidas em algumas regiões, através da implementação de políticas de extensão, assistência técnica, pesquisa agrícola e também merecendo aportes financeiros de programas agrícolas voltados para a produção agrícola sustentável (ASSAD; ALMEIDA, 2004).

Além dos aspectos técnicos, econômicos e sociais, os aspectos ambientais precisam ser observados para que se alcance a sustentabilidade, que Brandalise (2012) define como sendo o uso racional dos recursos para que possam ser repostos por processos naturais ou artificiais, respeitando sempre a capacidade regenerativa da natureza. Jackson (2013) concorda que para haver a prosperidade humana, deve existir o crescimento com o alcance de uma maior coesão social e maiores níveis de bem-estar, sempre buscando a redução de impactos sobre o meio ambiente, uma vez que seus recursos são finitos.

A sustentabilidade em uma propriedade rural, para Henriques e Richardson (2004), deve considerar o tripé da sustentabilidade: viabilidade econômica, consciência ambiental e responsabilidade social. 
Diante deste cenário, pretende-se entender o contexto da ruralidade no Brasil e os aspectos que envolvem a sustentabilidade. Assim, como pergunta de pesquisa tem-se: qual o panorama nas produções científicas brasileiras sobre o meio rural e os aspectos ligados à sustentabilidade?

Para este entendimento, objetiva-se analisar as produções científicas brasileiras sobre o meio rural e sobre aspectos da sustentabilidade. Para alcançar este objetivo, busca-se especificamente: (a) identificar os estudos publicados entre 2010 e 2015, no banco de teses e dissertações da Capes e os publicados entre 2006 e 2015 nas revistas brasileiras com Qualis/Capes A1 e A2 na área de Administração, contendo as palavras "rural" e "sustentabilidade" nos títulos e (b) analisar os principais indicadores científicos das produções.

Justifica-se o presente estudo pela necessidade de medir e avaliar as pesquisas científicas já existentes, de forma a permitir avaliar suas aplicabilidades, se e quando viáveis à realidade local ou permitir adaptações, quando necessárias. Adicionalmente, estudos que tiveram limitações na sua conclusão podem servir como um norteador quando aplicados a outras realidades ou, ainda, eventuais sugestões que tenham sido deixadas em outros trabalhos podem ser levados à realidade e à aplicação.

Price (1963) infere que os estudos das publicações científicas permitem vincular os conhecimentos, levando em conta quem realizou os estudos, bem como a evolução destes ao logo do tempo. Os conhecimentos e as práticas elaboradas pelos pesquisadores são incorporados a partir de um estudo bibliométrico, e tranformados em competências nos indivíduos (SANTOS, 2003).

\section{MATERIAL E MÉTODOS}

Para atingir os objetivos propostos neste estudo, realizou-se uma pesquisa exploratória, que segundo Honorato (2004), objetiva descobrir ideias, percepções e gerar hipóteses para um estudo mais aprofundado. Quanto aos procedimentos, utilizou-se a pesquisa bibliográfica, que Macedo (1994) define como sendo a busca por informações em documentos que se relacionam com o problema de pesquisa, 
sendo o primeiro passo para qualquer pesquisa científica, consistindo em uma "varredura" do que existe sobre o assunto e o conhecimento dos autores que tratam deste, a fim de que o autor não "reinvente a roda".

A coleta de dados ocorreu entre 28/03/2015 a 15/04/2015, contemplando os estudos publicados entre 2010 e 2015 , no banco de teses e dissertações da Capes e os estudos publicados entre os anos de 2006 a 2015 nas revistas brasileiras com Qualis/Capes A1 e A2 na área de Administração, contendo os termos "rural" e "sustentabilidade" nas palavras dos títulos. No Quadro 1 podem ser visualizados os periódicos pesquisados.

Quadro 1. Título das publicações pesquisadas

(continua)

\begin{tabular}{|l|c|}
\hline & Artigos \\
\hline 1 & BMC Cancer (Online) \\
\hline 2 & Brazilian journal of medical and biological research \\
\hline 4 & BAR. Brazilian Administration Review \\
\hline 5 & BBR. Brazilian Business Review (English Edition. Online) \\
\hline 6 & CERNE (UFLA) \\
\hline 7 & Ciência e Agrotecnologia (UFLA) \\
\hline 8 & Ciência Rural (UFSM. Impresso) \\
\hline 9 & Dados (Rio de Janeiro. Impresso) \\
\hline 10 & Engenharia Agrícola (Impresso) \\
\hline 11 & Engenharia Sanitária e Ambiental \\
\hline 12 & Estudos Avançados (USP. Impresso) \\
\hline 13 & Gestão \& Produção \\
\hline 14 & Gestão \& Produção (UFSCAR. Impresso) \\
\hline 15 & Opinião Pública (UNICAMP. Impresso) \\
\hline
\end{tabular}


(conclusão)

\begin{tabular}{|c|c|}
\hline & Artigos \\
\hline 16 & Organizações \& Sociedade (Online) \\
\hline 17 & Pesquisa Operacional (Impresso) \\
\hline 18 & Psicologia em Estudo (Impresso) \\
\hline 19 & Psicologia: Reflexão e Crítica (UFRGS. Impresso) \\
\hline 20 & Psicologia: Teoria e Pesquisa (UnB. Impresso) \\
\hline 21 & RAC Eletrônica \\
\hline 22 & RAC. Revista de Administração Contemporânea (Impresso) \\
\hline 23 & RAC. Revista de Administração Contemporânea (Online) \\
\hline 24 & RAE Eletrônica (Online) \\
\hline 25 & RAE (Impresso) \\
\hline 26 & RAE - Revista de Administração de Empresas \\
\hline 27 & RAUSP-e (São Paulo) \\
\hline 28 & Revista Árvore (Impresso) \\
\hline 29 & Revista Brasileira de Economia (Impresso) \\
\hline 30 & Revista Contabilidade \& Finanças (Impresso) \\
\hline 31 & Revista Contabilidade \& Finanças (Online) \\
\hline 32 & Revista de Administração Contemporânea \\
\hline 33 & Revista de Administração (FEA-USP) \\
\hline 34 & Revista de Administração Pública (Impresso) \\
\hline 35 & Revista de Administração (São Paulo. Online) \\
\hline 36 & Semina. Ciências Agrárias (Impresso) \\
\hline 37 & Semina. Ciências Agrárias (Online) \\
\hline 38 & Sociologias (UFRGS. Impresso) \\
\hline
\end{tabular}

Fonte: Dados da pesquisa (2015). 
Com os termos citados "rural" e "sustentabilidade", delimitou-se também a pesquisa nos títulos dos trabalhos para a busca das teses e dissertações, considerando os termos que aparecessem individualmente ou somados, sendo extraídas das seguintes áreas de conhecimento: Administração; Ciências ambientais; Sociais e humanidades; Engenharia de produção; Planejamento urbano e regional; Extensão rural; Extensão rural e desenvolvimento local; Economia; Desenvolvimento regional; Sociologia e Desenvolvimento sustentável.

Quanto aos critérios de exclusão dos trabalhos, ocorreu uma análise dos resumos das obras, excluíram-se da análise os trabalhos encontrados com o termo "rural" que não tinham relação com o meio rural somado a aspectos ligados a práticas administrativas e de gestão ou, quando das buscas pelo termo "sustentabilidade", que não estavam ligados ao meio rural.

A abordagem do problema é de caráter quantitativo, pois visa quantificar os dados, apresentando-os em tabelas, quadros e gráficos, e qualitativo, que Denzin e Lincoln (2006) definem como sendo o estudo que coleta uma variedade de materiais empíricos, podendo estes serem estudos de caso, experiências pessoais e outros exemplos, que descrevem momentos ou situações rotineiras na vida dos indivíduos, objetivando entender melhor o assunto que está ao seu alcance.

Os resultados são apresentados em quadros, tabelas e gráficos, visando uma melhor compreensão da revisão realizada, contemplando aspectos necessários ao cumprimento dos objetivos geral e específicos.

\section{RESULTADOS E DISCUSSÕES}

Os resultados são apresentados em duas seções. A primeira analisa os estudos de teses e dissertações, enquanto que a segunda, os artigos científicos.

O Quadro 2 apresenta um resumo das buscas ou pesquisas de trabalhos nas bases, constantes no objetivo, com suas respectivas datas, fontes de busca, palavraschave utilizadas, bem como as delimitações ou filtros utilizados para a busca e, adicionalmente, os resultados encontrados em cada situação. 
Quadro 2. Resumo das buscas de teses, dissertações e artigos científicos

\begin{tabular}{|c|c|c|c|c|}
\hline Data & Fonte & Palavra-chave & Delimitação & Resultados \\
\hline $15 / 04 / 2015$ & $\underline{\text { SciELO }}$ & Pequenos produtores rurais & Todos os índices & 0 \\
\hline $15 / 04 / 2015$ & $\underline{\text { SciELO }}$ & Assessoria administrativa & Todos os índices & 0 \\
\hline $15 / 04 / 2015$ & $\underline{\text { SciELO }}$ & $\begin{array}{c}\text { Gestão de pequenas } \\
\text { propriedades }\end{array}$ & Todos os índices & 0 \\
\hline $15 / 04 / 2015$ & $\underline{\text { SciELO }}$ & Sustentabilidade & Palavras do título & 273 \\
\hline $28 / 03 / 2015$ & Banco de Teses da Capes & Sustentabilidade & Título & 255 \\
\hline $12 / 04 / 2015$ & Banco de Teses da Capes & Rural & Título & 444 \\
\hline $11 / 04 / 2015$ & $\begin{array}{c}\text { Revistas - Qualis/Capes A1 } \\
\text { e A2 }\end{array}$ & Sustentabilidade & Título & 4 \\
\hline $11 / 04 / 2015$ & Revistas - Qualis/Capes A1 & Rural & Título & 9 \\
\hline
\end{tabular}

Fonte: Dados da pesquisa (2015).

Iniciou-se a busca por quatro palavras-chave, sendo: "pequenos produtores rurais"; "assessoria administrativa"; "gestão de pequenas propriedades"; e "sustentabilidade". Optou-se por este grupo de palavras, inicialmente, com vistas a identificar o estado da arte das publicações sobre a administração aplicada principalmente nas pequenas propriedades rurais.

No início, para se ter uma ideia sobre o panorama e quantidade de publicações existentes, utilizou-se a base de dados $S c i E L O$, na qual ocorreu a pesquisa das palavras "pequenos produtores rurais"; "assessoria administrativa"; "gestão de pequenas propriedades", permitindo a busca em todos os índices, porém, ambas com nenhum resultado. Já para a palavra-chave "sustentabilidade", delimitando-se a busca apenas nas palavras dos títulos dos trabalhos, surgiram 273 resultados, com estudos que abordavam a questão da sustentabilidade, porém não só com foco no meio rural, mas em todos os setores ou atividades.

Logo, decidiu-se pela substituição das três primeiras palavras-chave pelo termo "rural", mantendo a palavra-chave "sustentabilidade". Desta forma, iniciou-se a pesquisa pela base de teses e dissertações da Capes, onde se encontrou, para o termo "sustentabilidade", 255 trabalhos e para o termo "rural" outros 444 estudos, delimitando a pesquisa apenas nas palavras dos títulos. Destes, 09 trabalhos com 
o termo "sustentabilidade" foram escolhidos, pois através da leitura dos resumos das obras, identificou-se que as mesmas tinham relação com o objetivos, ou seja, que estavam ligadas ao meio rural e a aspectos ligados às práticas administrativas e de gestão, dos quais apenas 05 estavam disponíveis para a leitura integral ou para download, mantendo-se apenas estes para a continuidade do estudo. Com o termo "rural", 12 trabalhos foram inicialmente selecionados, dos quais 09 estavam disponíveis e, por este motivo, foram escolhidos, totalizando 14 trabalhos.

Com relação aos artigos científicos, as mesmas palavras-chave foram utilizadas, sendo que a busca ocorreu nas palavras dos títulos dos estudos, em todas as revistas brasileiras, com avaliação Qualis/Capes A1 e A2 para Administração. Nestes critérios, optou-se por 13 trabalhos que estavam de acordo com a proposta central.

\subsection{ANÁLISES DAS DISSERTAÇÕES DE MESTRADO E TESES DE DOUTORADO}

O Quadro 3 mostra a distribuição dos trabalhos encontrados, por nível de especialização e por ano de publicação. Destacam-se as publicações em nível de mestrado no ano de 2011, com 08 trabalhos.

Quadro 3. Distribuição dos trabalhos por nível de especialização e ano de publicação

\begin{tabular}{|l|c|c|c|c|}
\hline Nível & $\mathbf{2 0 1 1}$ & $\mathbf{2 0 1 2}$ & $\mathbf{2 0 1 3}$ & Total \\
\hline Doutorado & 0 & 2 & 0 & 2 \\
\hline Mestrado & 8 & 3 & 1 & 12 \\
\hline Total & $\mathbf{8}$ & $\mathbf{5}$ & $\mathbf{1}$ & $\mathbf{1 4}$ \\
\hline
\end{tabular}

Fonte: Dados da pesquisa (2015).

O Quadro 4 apresenta a distribuição dos estudos por origem dos autores. Destacam-se a Universidade Federal de Pernambuco (UFPE) e a Universidade Federal Rural de Pernambuco (UFRPE), com duas dissertações cada. Ao analisar os estudos por área geográfica, no Nordeste desenvolveram-se 05 estudos; no Sul e Sudeste, 03 estudos em cada uma das regiões, enquanto que no Centro Oeste, 02 estudos. 
Quadro 4. Origem dos autores

\begin{tabular}{|l|l|c|c|}
\hline $\begin{array}{l}\text { Origem do autor } \\
\text { (I.E.) }\end{array}$ & Nome da instituição de ensino (I.E.) & Estado & Quantidade \\
\hline Nível: Doutorado & & $\mathbf{2}$ & 1 \\
\hline UFU & Universidade Federal de Uberlândia & MG & SP \\
\hline UNINOVE & Universidade Nove de Julho & & $\mathbf{1 2}$ \\
\hline Nível: Mestrado & & PE & 2 \\
\hline UFPE & Universidade Federal de Pernambuco & PE & 2 \\
\hline UFRPE & Universidade Federal Rural de Pernambuco & MG & 1 \\
\hline FPL & Fundação Pedro Leopoldo & GO & 1 \\
\hline PUC & Pontifícia Universidade Católica & MS & 1 \\
\hline UFMS & Universidade Federal de Mato Grosso do Sul & PB & 1 \\
\hline UFPB & Universidade Federal da Paraíba & RS & 1 \\
\hline UFPEL & Universidade Federal de Pelotas & RS & 1 \\
\hline UNISC & Universidade de Santa Cruz do Sul & SC & 1 \\
\hline UNOCHAPECÓ & Universidade Comunitária Regional de Chapecó & SP & 1 \\
\hline USP & Universidade de São Paulo & & $\mathbf{1 4}$ \\
\hline Total & & & \\
\hline
\end{tabular}

Fonte: Dados da pesquisa (2015).

Os autores que se voltam para o estudo da temática escolhida para este artigo estão relacionados no Quadro 5, que evidencia os nomes dos autores, acompanhado do ano da defesa, bem como dos títulos das respectivas obras. 
Quadro 5. Autores, ano de defesa e título das obras

\begin{tabular}{|c|c|}
\hline $\begin{array}{l}\text { Autor e ano de } \\
\text { publicação }\end{array}$ & Títulos das obras \\
\hline Martins (2011) & $\begin{array}{l}\text { Indicadores de desenvolvimento rural para os territórios CONSAD de Mato } \\
\text { Grosso do Sul. (Dissertação) }\end{array}$ \\
\hline Lim & $\begin{array}{l}\text { A agroecologia e extensão rural para o fortalecimento da agricultura } \\
\text { familiar: o caso do município de Santa Cruz da Baixa Verde - Pernambuco. } \\
\text { (Dissertação) }\end{array}$ \\
\hline Pinheiro (2011) & $\begin{array}{l}\text { Avaliação da sustentabilidade de sistemas de produção apícolas - diagnóstico } \\
\text { participativo em associações de apicultores da região central do Ceará. } \\
\text { (Dissertação) }\end{array}$ \\
\hline Eusébio (2011) & $\begin{array}{l}\text { Determinantes do acesso ao crédito rural: um estudo a partir do levantamento } \\
\text { das unidades produtivas agropecuárias (LUPA) do estado de São Paulo. } \\
\text { (Dissertação) }\end{array}$ \\
\hline Silva & $\begin{array}{l}\text { Agricultura familiar e arranjos produtivos locais no Semiárido alagoano: } \\
\text { possibilidades e limites para a extensão rural na perspectiva do } \\
\text { desenvolvimento local. (Dissertação) }\end{array}$ \\
\hline Botelho (2011) & $\begin{array}{l}\text { A política de assistência técnica e extensão rural para os processos de } \\
\text { desenvolvimento local em Pernambuco: um estudo analítico. (Dissertação) }\end{array}$ \\
\hline Nino & $\begin{array}{l}\text { O conflito sobre os usos da propriedade rural face os imperativos da } \\
\text { legislação ambiental: estudo sobre as representações de atores sociais acerca } \\
\text { da questão das áreas de preservação permanente e reserva legal/Pelotas, RS. } \\
\text { (Dissertação) }\end{array}$ \\
\hline Aguiar (2011) & $\begin{array}{l}\text { Agricultura familiar: desafios para a sustentabilidade socioeconômica e } \\
\text { ambiental. (Dissertação) }\end{array}$ \\
\hline Aléssio (2012) & $\begin{array}{l}\text { Desenvolvimento e sustentabilidade no Oeste Catarinense: contradições, } \\
\text { consensos e alternativas. (Dissertação) }\end{array}$ \\
\hline $\begin{array}{l}\text { Machado Junior } \\
\text { (2012) }\end{array}$ & $\begin{array}{l}\text { A influência de pesquisadores do strictu sensu em Administração na } \\
\text { legitimação do conhecimento em sustentabilidade ambiental. (Tese) }\end{array}$ \\
\hline Marques (2012) & $\begin{array}{l}\text { A sustentabilidade de unidades de produção familiares nos assentamentos de } \\
\text { reforma agrária do sertão pernambucano. (Dissertação) }\end{array}$ \\
\hline Silva (2012) & $\begin{array}{l}\text { A agricultura familiar e a função social da propriedade rural: o caso da região } \\
\text { fronteira oeste do Rio Grande do Sul. (Dissertação) }\end{array}$ \\
\hline Queiroz (2012) & $\begin{array}{l}\text { PRONAF e desenvolvimento rural sustentável: uma análise econométrica } \\
\text { espacial dos impactos do programa nas regiões Sul e Nordeste, do Brasil, } \\
\text { entre } 2000 \text { e } 2006 \text {. (Tese) }\end{array}$ \\
\hline Souza (2013) & $\begin{array}{l}\text { Sistemas de informações gerenciais no agronegócio: estudo de caso de } \\
\text { aplicação de software em Administração Rural pelos produtores de grãos do } \\
\text { município de Rio Verde - GO. (Dissertação) }\end{array}$ \\
\hline
\end{tabular}

Fonte: Dados da pesquisa (2015). 
Nas obras, 54 palavras-chave foram citadas, em que há destaque para os termos "agricultura familiar", com 05 citações, "sustentabilidade" com 04 , seguida de "extensão rural" com 03 aparições e "desenvolvimento local", "crédito rural" e "desenvolvimento rural" com 02 citações cada. As demais aparecem 01 vez como se visualiza no Quadro 6.

Quadro 6. Palavras-chave das dissertações e teses

(continua)

\begin{tabular}{|l|c|}
\hline Palavra-chave & Quantidade \\
\hline Agricultura familiar & 5 \\
\hline Sustentabilidade & 4 \\
\hline Extensão rural & 2 \\
\hline Desenvolvimento local & 2 \\
\hline Crédito rural & 2 \\
\hline Desenvolvimento rural & 1 \\
\hline Apicultura & 1 \\
\hline Política pública & 1 \\
\hline Indicadores agropecuários e econometria espacial & 1 \\
\hline Arranjos produtivos & 1 \\
\hline Representações & 1 \\
\hline Árvore de regressão e classificação & 1 \\
\hline Função social & 1 \\
\hline Assistência técnica & 1 \\
\hline Manejo florestal & 1 \\
\hline CONSAD & 1 \\
\hline Propriedade & 1 \\
\hline Convivência com o semi-árido & 1 \\
\hline Sistema de informação gerencial & 3 \\
\hline Administração rural & 1 \\
\hline
\end{tabular}


(conclusão)

\begin{tabular}{|l|c|}
\hline Palavra-chave & Quantidade \\
\hline Análise de redes sociais & 1 \\
\hline Crescimento econômico & 1 \\
\hline Indicadores & 1 \\
\hline Data-mining & 1 \\
\hline Institucionalização do conhecimento & 1 \\
\hline Desenvolvimento & 1 \\
\hline Oeste Catarinense & 1 \\
\hline Agronegócio & 1 \\
\hline PRONAF & 1 \\
\hline Desenvolvimento regional & 1 \\
\hline Propriedade rural & 1 \\
\hline Sustentabilidade ambiental & 1 \\
\hline Reserva Legal & 1 \\
\hline Tecnologia da informação & 1 \\
\hline Software de gerenciamento rural & 1 \\
\hline Trevo da sustentabilidade & 1 \\
\hline Áreas de preservação permanente & 1 \\
\hline Ensino e pesquisa em Administração & 1 \\
\hline Transição agroecológica & $\mathbf{1 4}$ \\
\hline Desenvolvimento sustentável & 1 \\
\hline Acesso ao crédito & 1 \\
\hline Diagnóstico & 1 \\
\hline Total & 1 \\
\hline & 1 \\
\hline
\end{tabular}

Fonte: Dados da pesquisa (2015).

No Quadro 7 estão elencadas as sugestões para futuros estudos, de acordo com 05 autores. 
Quadro 7. Sugestões que emergiram a partir das obras analisadas

\begin{tabular}{|l|l|}
\hline \multicolumn{1}{|c|}{$\begin{array}{l}\text { Autor e ano } \\
\text { de publicação }\end{array}$} & \multicolumn{1}{c|}{ Sugestão } \\
\hline Martins (2011) & $\begin{array}{l}\text { Construção de indicadores de desenvolvimento rural, seja por meio da escolha de outras } \\
\text { variáveis, atualização dos dados utilizados nesta pesquisa ou por meio da aplicação de outros } \\
\text { modelos que evidenciem o grau de desenvolvimento rural de outras regiões ou contextos, } \\
\text { tendo em vista a importância do tema atualmente. }\end{array}$ \\
\hline $\begin{array}{l}\text { Pinheiro } \\
\text { (2011) }\end{array}$ & $\begin{array}{l}\text { Avaliação de assistência técnica em projetos de investimentos governamentais; avaliação } \\
\text { dos programas governamentais de apoio à apicultura na região da caatinga; aplicação da } \\
\text { metodologia Trevo da Sustentabilidade em outros conglomerados apícolas na região } \\
\text { central do Ceará, investigando os motivos que levam os apicultores a se aproximarem ou se } \\
\text { afastarem de um desenvolvimento sustentável. }\end{array}$ \\
\hline $\begin{array}{l}\text { Machado } \\
\text { Junior (2012) }\end{array}$ & $\begin{array}{l}\text { Recomenda-se que estudos futuros estabeleçam um recorte de investigação da etapa de } \\
\text { objetivação, visando identificar quais os métodos, as abordagens teóricas, os temas e outras } \\
\text { características predominantes, no que tange às pesquisas em sustentabilidade ambiental. } \\
\text { Adicionalmente, recomenda-se que a revisão destes estudos utilize uma abordagem } \\
\text { qualitativa, visando à investigação do conteúdo das teses e dissertações, com uma análise da } \\
\text { evolução da estrutura de colaboração gerada pelos laços de participação, ao longo de quatro } \\
\text { triênios de análise. }\end{array}$ \\
\hline $\begin{array}{l}\text { Marques } \\
(2012)\end{array}$ & $\begin{array}{l}\text { A dissertação objetivou avaliar a sustentabilidade de unidades de produção familiares } \\
\text { no Semiárido pernambucano, através de indicadores econômicos, sociais e ambientais, } \\
\text { constituindo um banco de dados inédito, sendo que a pesquisa realizada continua em } \\
\text { andamento e sugerindo uma tese de doutorado. }\end{array}$ \\
\hline Souza (2013) & $\begin{array}{l}\text { Sugere-se a realização de outros estudos em outros departamentos e estágios do } \\
\text { agronegócio, sobre o uso de software agrícola, bem como realizar em outros Estados e } \\
\text { cidades, permitindo comparações e, não obstante, realizar estudos centrados unicamente } \\
\text { no funcionário das empresas rurais. }\end{array}$ \\
\hline
\end{tabular}

Fonte: Dados da pesquisa (2015).

De acordo com estas sugestões, percebe-se que os métodos e técnicas podem ser empregados em outros contextos para a verificação de resultados e possíveis comparações. Assim, os assuntos abordados por parte destes autores oferecem um direcionamento para novos pesquisadores, o que para a comunidade acadêmica em nível de graduação ou especialização latu sensu ou strictu sensu constitui-se em uma importante contribuição.

\subsection{ANÁLISES DOS ARTIGOS CIENTÍFICOS}

Junto às revistas com avaliação Qualis/Capes em A1 e A2, houve a pesquisa individual por revista, utilizando-se as duas palavras-chave: "rural" e "sustentabilidade", em que a mesma apareceu nas palavras dos títulos dos artigos. 
Desta forma, identificou-se 13 artigos, os quais, através de uma breve análise de conteúdo, estavam de acordo com o objetivo da pesquisa. Os demais artigos encontrados com as palavras-chave, que continham em seus títulos uma ou ambas as palavras, mas que, através da leitura de seus resumos, não estavam de acordo com o tema do presente estudo, foram excluídos, sendo que a maioria destes ocorreu para o termo "sustentabilidade", pois o termo de forma isolada gerou um grande número de resultados, mas a questão abordada não estava ligada ao meio rural. No Quadro 8, podem ser visualizados os autores e o título dos artigos selecionados para a revisão e consequente construção deste estudo.

Quadro 8. Autores, ano de publicação e título dos artigos

\begin{tabular}{|c|c|}
\hline $\begin{array}{l}\text { Heidtmann Junior e } \\
\text { Loch (2014) }\end{array}$ & A paisagem cultural e as novas possibilidades para a atividade familiar rural. \\
\hline $\begin{array}{l}\text { Ribeiro, Andion e } \\
\text { Burigo (2015) }\end{array}$ & $\begin{array}{l}\text { Ação coletiva e coprodução para o desenvolvimento rural: um estudo de caso do } \\
\text { Colegiado de Desenvolvimento Territorial da Serra Catarinense. }\end{array}$ \\
\hline $\begin{array}{l}\text { Kunzler e Bulgacov } \\
\text { (2011) }\end{array}$ & $\begin{array}{l}\text { As estratégias competitivas e colaborativas e os resultados individuais e coletivos do } \\
\text { associativismo rural em Quatro Pontes (PR). }\end{array}$ \\
\hline $\begin{array}{l}\text { Capellesso e Cazella } \\
\text { (2013) }\end{array}$ & $\begin{array}{l}\text { Indicador de sustentabilidade dos agroecossistemas: estudo de caso em áreas de } \\
\text { cultivo de milho. }\end{array}$ \\
\hline $\begin{array}{l}\text { Carneiro Neto et al. } \\
\text { (2008) }\end{array}$ & Índice de sustentabilidade agroambiental para o perímetro irrigado Ayres de Souza. \\
\hline $\begin{array}{l}\text { Freitas, Freitas e Dias } \\
(2012)\end{array}$ & $\begin{array}{l}\text { Mudanças conceituais do desenvolvimento rural e suas influências nas políticas } \\
\text { públicas. }\end{array}$ \\
\hline Rosalen (2014) & The impact of the Law $10,267 / 2001$ in the brazilian rural registration \\
\hline Landini (2014) & $\begin{array}{l}\text { Problemas enfrentados por extensionistas rurais brasileiros e sua relação com suas } \\
\text { concepções de extensão rural. }\end{array}$ \\
\hline $\begin{array}{l}\text { Marinho e Araújo } \\
(2010)\end{array}$ & Pobreza e o sistema de seguridade social rural no Brasil. \\
\hline Vizeu (2011) & Rural heritage of early brazilian industrialists: its impact on managerial orientation. \\
\hline $\begin{array}{l}\text { Pires, Barbosa e } \\
\text { Albuquerque (2013) }\end{array}$ & $\begin{array}{l}\text { Sustentabilidade de empreendimentos econômicos solidários: análise da cooperativa } \\
\text { de fruticultores de Abaetetuba. }\end{array}$ \\
\hline $\begin{array}{l}\text { Vernetti Junior, } \\
\text { Gomes e Schuch } \\
(2009)\end{array}$ & $\begin{array}{l}\text { Sustentabilidade de sistemas de rotação e sucessão de culturas em solos de várzea no } \\
\text { sul do Brasil. }\end{array}$ \\
\hline Van Der Ploeg (2011) & Trajetórias do desenvolvimento rural: pesquisa comparativa internacional. \\
\hline
\end{tabular}

Fonte: Dados da pesquisa (2015). 
Nenhum artigo foi encontrado nas revistas com Qualis A1, contudo, 13 artigos foram selecionados das revistas com Qualis A2, conforme se vê no Quadro 9.

Quadro 9. Quantidade de artigos científicos por classificação Qualis/Capes

\begin{tabular}{|l|r|}
\hline Classificação Qualis/CAPES & Quantidade \\
\hline A1 & 0 \\
\hline A2 & 13 \\
\hline Total & $\mathbf{1 3}$ \\
\hline
\end{tabular}

Fonte: Dados da pesquisa (2015).

Dos 13 trabalhos encontrados, 04 foram publicados na Revista de Administração Pública (Impresso) e outros 04 artigos da Revista Ciência Rural (UFSM - Impresso). Os demais artigos foram encontrados em 05 revistas diferentes, conforme mostra o Quadro 10.

Quadro 10. Distribuição dos artigos por título de periódico científico

\begin{tabular}{|l|c|}
\hline Revista & $\mathbf{n}^{\mathbf{0}}$ publicações \\
\hline Revista de Administração Pública & 4 \\
\hline Ciência Rural & 4 \\
\hline Revista Brasileira de Economia & 1 \\
\hline Ciência e Agrotecnologia & 1 \\
\hline Sociologias & 1 \\
\hline Brazilian Administration Review (BAR) & 1 \\
\hline Engenharia agrícola & 1 \\
\hline Total & $\mathbf{1 3}$ \\
\hline
\end{tabular}

Fonte: Dados da pesquisa (2015).

No que tange ao período das publicações, observou-se dois picos: um em 2014, com 03 publicações e outros 03 em 2011, sendo que o primeiro estudo encontrado foi publicado em 2008 e o último no ano de 2015, a considerar que esta revisão de estudos tem como período de análise para os artigos científicos o prazo de 10 anos, ou seja, desde 2006. Estes dados podem ser visualizados no Quadro 11. 
Quadro 11. Distribuição dos artigos por ano de publicação

\begin{tabular}{|l|c|}
\hline Ano de publicação & Quantidade \\
\hline 2014 & 3 \\
\hline 2011 & 3 \\
\hline 2013 & 2 \\
\hline 2015 & 1 \\
\hline 2012 & 1 \\
\hline 2010 & 1 \\
\hline 2009 & 1 \\
\hline 2008 & 1 \\
\hline Total geral & $\mathbf{1 3}$ \\
\hline
\end{tabular}

Fonte: Dados da pesquisa (2015).

Para os 13 artigos há 29 autores, quando somados todos os que estavam envolvidos. Destes, 04 artigos foram publicados por 01 autor; 04 artigos por 02 autores; 04 artigos por 03 autores e 01 por 05 autores.

Quanto à origem dos autores, as instituições de ensino UFC, UFPA, UFSC, UDESC e UFV, bem como a EMBRAPA, mereceram destaque quanto à quantidade de estudos publicados por autores advindos destas instituições, conforme se observa no Quadro 12.

Quadro 12. Distribuição dos artigos por instituição de ensino ou fora do contexto acadêmimo

(continua)

\begin{tabular}{|l|c|}
\hline Instituição & Quantidade \\
\hline UFC - Universidade Federal do Ceará & 5 \\
\hline EMBRAPA - Empresa Brasileira de Pesquisa Agropecuária & 3 \\
\hline UFPA - Universidade Federal do Pará & 3 \\
\hline UFSC - Universidade Federal de Santa Catarina & 3 \\
\hline UDESC - Universidade do Estado de Santa Catarina & 2 \\
\hline
\end{tabular}


(conclusão)

\begin{tabular}{|l|c|}
\hline Instituição & Quantidade \\
\hline UFV - Universidade Federal de Viçosa & 2 \\
\hline EPAGRI - Empresa de Pesquisa Agropecuária e Extensão Rural de Santa Catarina & 1 \\
\hline FGV/SP - Fundação Getúlio Vargas/São Paulo & 1 \\
\hline IFSC - Instituto Federal de Santa Catarina & 1 \\
\hline UBA - Universidad de Buenos Aires - Argentina & 1 \\
\hline UFMG - Universidade Federal de Minas Gerais & 1 \\
\hline UFPel - Universidade Federal de Pelotas & 1 \\
\hline UNESP - Universidade Estadual Paulista & 1 \\
\hline UNIOESTE - Universidade Estadual do Oeste do Paraná & 1 \\
\hline Universidade de Wageningen - Holanda / Universidade Agrícola de Beijing - China & 1 \\
\hline Universidade Positivo & 1 \\
\hline $\begin{array}{l}\text { COGERH - Companhia de Gestão dos Recursos Hídricos - Governo do Estado do } \\
\text { Ceará }\end{array}$ & 1 \\
\hline Total & $\mathbf{2 9}$ \\
\hline
\end{tabular}

Fonte: Dados da pesquisa (2015).

Geograficamente, 09 trabalhos vieram de instituições do Sul do Brasil; 05 trabalhos do Nordeste e 05 do Sudeste; 03 da região Norte; e, ainda, 02 artigos de autores de instituições do exterior, como a UBA, de Buenos Aires - Argentina e mais um autor atuante em duas Universidades, sendo a primeira Universidade de Wageningen - Holanda e Universidade Agrícola de Beijing - China. Adicionalmente, 05 autores são de instituições externas ao contexto acadêmico. A distribuição dos artigos pode ser analisada pelo Quadro 13. 
Quadro 13. Distribuição dos artigos por autores versus região de localização

\begin{tabular}{|l|c|}
\hline Localização & Quantidade \\
\hline Sul do Brasil & 9 \\
\hline Nordeste do Brasil & 5 \\
\hline Sudeste do Brasil & 5 \\
\hline Instituições externas & 5 \\
\hline Norte do Brasil & 3 \\
\hline Exterior & 2 \\
\hline Total & 29 \\
\hline
\end{tabular}

Fonte: Dados da pesquisa (2015).

Dos artigos, 02 tratam de calcular indicadores de sustentabilidade, enquanto que 09 do total tratam de assuntos ligados ao processo de desenvolvimento rural, abordando aspectos inerentes a este processo. Os 04 estudos restantes tratam de situações ou problemas enfrentados pelos proprietários rurais, advindos de forças externas ou pelos extensionistas, no que tange à assistência aos agricultores, podendo ser, desta forma, categorizados os artigos catalogados para esta obra.

\section{CONSIDERAÇÕES FINAIS}

Ao analisar as pesquisas científicas brasileiras sobre o meio rural e sobre aspectos da sustentabilidade, no período entre 2010 e 2015, no banco de teses e dissertações da Capes e revistas brasileiras com Qualis/Capes A1 e A2 na área de Administração, no período de 2006 a 2015, conclui-se que são poucos os estudos que se voltam ao tema, principalmente quando o aspecto principal a ser considerado é o meio rural e práticas administrativas e de gestão neste segmento.

Neste sentido, foram poucos também os estudos que puderam ser utilizados para a execução desta revisão bibliográfica, contudo, foi possível traçar um panorama acerca do estado da arte neste tema.

A maioria dos estudos concentrou-se no Nordeste, quando se analisou as teses e dissertações da base da Capes e, quando analisados os artigos, o Sul teve a 
maior representatividade, seguido da região Nordeste e Sudeste. Neste aspecto, as duas regiões concentram uma grande quantidade de pequenas propriedades rurais e de agricultores familiares, que lutam primordialmente para o sustento da família. Assim, os estudos buscaram entender este contexto e auxiliar com as referidas pesquisas, na melhoria das condições.

Nenhum artigo foi encontrado nas revistas brasileiras com Qualis/Capes A1 e A2, com o uso das palavras-chave "rural" e "sustentabilidade", o que sugere a falta de interesse dos pesquisadores, principalmente do meio acadêmico e, também, dos editores e avaliadores das revistas, o que precisa ser revisto, uma vez que o Brasil tem suas origens a partir da produção agrícola, com a extração do Pau-Brasil e, hoje, tem como uma das principais atividades geradoras de riqueza o agronegócio.

Para pesquisas futuras, sugere-se a continuidade da revisão bibliográfica neste tema, abrangendo outras bases de dados, tais como SciELO, Elsevier, Emerald, JSTOR, EBSCO, ScienceDirect, além dos anais da AnPad e outros eventos bem conceituados pelo meio acadêmico.

\section{REFERÊNCIAS}

AGUIAR, V. M. Q. F. Agricultura familiar: desafios para a sustentabilidade socioeconômica e ambiental. 164f. Dissertação (Mestrado em Direito) - Programa de Pós-Graduação em Direito, Relações Internacionais e Desenvolvimento, Pontifícia Universidade Católica de Goiás, Goiânia, 2011.

ALÉSSIO, B. C. Desenvolvimento e sustentabilidade no oeste catarinense: contradições, consensos e alternativas. 2012. 144f. Dissertação (Mestrado) Universidade Comunitária da Região de Chapecó, UNOCHAPECÓ, 2012. 144p.

ASSAD, M. L. L.; ALMEIDA, J. Agricultura e sustentabilidade. Contexto, desafios e cenários. Ciência \& Ambiente. São Paulo, v. 1, n. 294, p. 15-30, abr. 2004.

BRANDALISE, L. T. Desenvolvimento sustentável e o setor público. In: BRANDALISE, L. T.; NAZZARI, R. K. (Org.) Políticas de sustentabilidade. Responsabilidade social e corporativa das questões ecológicas. Cascavel: EDUNIOESTE, 2012. Cap. 2, p. 16-68. 
BOTELHO, L. C. A política de assistência técnica e extensão rural para os processos de desenvolvimento local em Pernambuco: um estudo analítico. 2011. 98f. Dissertação (Mestrado em Extensão Rural e Desenvolvimento Local) Universidade Federal Rural de Pernambuco, 2011.

CAPELLESSO,A.J.;CAZELLA,A.A. Indicador de sustentabilidade dos agroecossistemas: estudo de caso em áreas de cultivo de milho. Ciência Rural, v. 43, n. 12, p. 2297 2303, 2013.

CARNEIRO NETO, J. A. C. et al. Índice de sustentabilidade agroambiental para o perímetro irrigado Ayres de Souza. Ciência e Agrotecnologia, v. 32, n. 4, p. 12721279, 2008.

CARVAlHO, C. de O.; SANTOS, A. C. dos; CARVAlHO, G. R. Rede Brasil Rural: Inovação no Contexto da Agricultura Familiar. Revista em Agronegócio e Meio Ambiente, v. 8, n. 1, p. 79-94, jan./abr. 2015.

DENZIN, N. K.; LINCOLN, Y. S. O planejamento da pesquisa qualitativa: teorias e abordagens. Tradução de Sandra Regina Netz. Porto Alegre: Artmed, 2006.

EUSÉBIO, G. S. Determinantes do acesso ao crédito rural: um estudo a partir do levantamento das unidades produtivas agropecuárias (LUPA) do Estado de São Paulo. 2011. 112f. Dissertação (Mestrado) - Faculdade de Economia, Administração e Contabilidade de Ribeirão Preto, Universidade de São Paulo, Ribeirão Preto, 2011.

FREITAS, A. F. de; FREITAS, A. F. de; DIAS, M. M. Mudanças conceituais do desenvolvimento rural e suas influências nas políticas públicas. RAP: Revista Brasileira de Administração Pública, v. 46, n. 6, 2012.

HEIDTMANNJUNIOR, D.E. D.; LOCH, C. A paisagem cultural e as novas possibilidades para a atividade familiar rural. Ciência Rural, v. 44, n. 11, p. 1988-1994, 2014.

HENRIQUES, A.; RICHARDSON, J. Introduction: Triple Bottom Line - Does it all add up? In: HENRIQUES, A.; RICHARDSON, J. (Ed.). The triple bottom line: does it all add up? Assessing the sustainability of business and CSR. Earthscan: London, 2004. p. ixx-xxii. 
HONORATO, G. Conhecendo o marketing. Barueri: Manole, 2004.

JACKSON, T. Prosperidade sem crescimento: vida boa em um planeta finito. São Paulo: Planeta Sustentável; Ed. Abril, 2013.

KUMMER, L. Metodologia participativa no meio rural: uma visão interdisciplinar. Conceitos, ferramentas e vivências. Salvador: GTZ, 2007.

KUNZLER, M. T.; BULGACOV, S. As estratégias competitivas e colaborativas e os resultados individuais e coletivos no associativismo rural em Quatro Pontes (PR). Revista de Administração Pública. Rio de Janeiro, v. 45, n. 5, p. 363-393, 2011.

LAMARCHE, H. Agricultura familiar: uma realidade multiforme. Campinas: Ed. UNICAMP, 1993.

LANDINI, F. P. Problemas enfrentados por extensionistas rurais brasileiros e sua relação com suas concepções de extensão rural. Ciência Rural, v. 45, n. 2, 2015.

LIMA, F. A. X. A agroecologia e extensão rural para o fortalecimento da agricultura familiar: o caso do município de Santa Cruz da Baixa Verde - Pernambuco. 2011. $143 \mathrm{f}$. Dissertação (Mestrado em Extensão Rural e Desenvolvimento Local) - Universidade Federal Rural de Pernambuco, 2011.

MACEDO, N. D. de. Iniciação à pesquisa bibliográfica: guia do estudante para a fundamentação do trabalho de pesquisa. 2. ed. revista. São Paulo: Loyola, 1994.

MACHADO JUNIOR, C. A influência de pesquisadores do stricto sensu em Administração na legitimação do conhecimento em sustentabilidade ambiental. Tese (Doutorado em Administração) - Universidade Nove de Julho, 2012.

MARINHO, E.; ARAUJO, J. Pobreza e o sistema de seguridade social rural no Brasil. Revista Brasileira de Economia, v. 64, n. 2, p. 161-174, 2010.

MARQUES, M. W. C. da F. A sustentabilidade de unidades de produção familiares 
nos assentamentos de reforma agrária do sertão pernambucano. 2012. $104 \mathrm{f}$. Dissertação (Mestrado em Administração e Desenvolvimento Rural) - Universidade Federal Rural de Pernambuco, 2012.

MARTINS, C. L. Indicadores de desenvolvimento rural para os territórios CONSAD de Mato Grosso do Sul. 2011. 109f. Dissertação (Mestrado em Administração) - Universidade Federal de Mato Grosso do Sul, Campo Grande, 2011.

NINO, L. B. O Conflito sobre os usos da propriedade rural face os imperativos da legislação ambiental: estudo sobre as representações de atores sociais acerca da questão das áreas de preservação permanente e reserva legal/Pelotas, RS. 2011. $193 \mathrm{f}$. Dissertação (Mestrado) - Universidade Federal de Pelotas, Pelotas, 2011.

PINHEIRO, F. K. Avaliação da sustentabilidade de sistemas de produção apícolas - diagnóstico participativo em associações de apicultores da região central do Ceará. 2011. 168f. Dissertação (Mestrado em Engenharia de Produção) - Universidade Federal da Paraíba, 2011.

PIRES, A. R. S.; BARBOSA, M. J. de S.; ALBUQUERQUE, F. dos S. Sustentabilidade de empreendimentos econômicos solidários: análise da cooperativa dos fruticultores de Abaetetuba. RAP: Revista Brasileira de Administração Pública, v. 47, n. 5, 2013.

PRICE, D. de S. Big science, little science. Columbia University, New York, p. 119119, 1963.

QUEIROZ, S. F. de. PRONAF e desenvolvimento rural sustentábel: uma análise econométrica espacial dos impactos do programa nas regiões Sul e Nordeste, do Brasil, entre 2000 e 2006. 258f. Tese (Doutorado em Economia) - Universidade Federal de Uberlândia, 2012.

REDE NACIONAL DE INFORMAÇÕES SOBRE O INVESTIMENTO - RENAI. Por que Brasil? Disponível em: < http://investimentos.desenvolvimento.gov.br/>. Acesso em: 29 set. 2014. 
ROESLER, D. A. Racionalidades e práticas administrativas do agricultor familiar no contexto ambiental: um estudo em três comunidades rurais do Sul da região metropolitana de Curitiba - Paraná. 2009. 327f. (Tese de Doutorado) - Universidade Federal do Paraná - UFPR, Curitiba, 2009.

ROSALEN, D. L. The impact of the law $10,267 / 2001$ in the brazilian rural registration. Engenharia Agrícola, v. 34, n. 2, p. 372-384, 2014.

RIBEIRO, A. C.; ANDION, C.; BURIGO, F. Ação coletiva e coprodução para o desenvolvimento rural: um estudo de caso do Colegiado de Desenvolvimento Territorial da Serra Catarinense. Revista de Administração Pública, v. 49, n. 1, p. 119-140, 2015.

SANTOS, G. J. dos; MARION, J. C.; SEGATTI, S. Administração de custos na agropecuária. 2. ed. São Paulo: Atlas, 1996.

SANTOS, R. N. M. dos. Produção científica: por que medir? o que medir?. Revista digital de Biblioteconomia e Ciência da Informação, v. 1, n. 1, 2003.

SILVA, J. R. da. Agricultura familiar e arranjos produtivos locais no semiárido alagoano: possibilidades e limites para a extensão rural na perspectiva do desenvimento local. 2011. 116f. Dissertação (Mestrado em Extensão Rural e Desenvolvimento Local) - Universidade Federal Rural de Pernambuco, 2011.

SILVA, P. T. O. da. A agricultura familiar e a função social da propriedade rural: o caso da região fronteira Oeste do Rio Grande do Sul. 2012. 130f. Dissertação. (Mestrado em Desenvolvimento Regional) - Universidade de Santa Cruz do Sul, 2012 .

SOUZA, S. dos S. Sistemas de informações gerenciais no agronegócio: estudo de caso de aplicação do software em administração rural pelos produtores de grãos do município de Rio Verde - GO. 2013. 182f. Dissertação (Mestrado Profissional em Administração) - Fundação Cultural Dr. Pedro Leopoldo, 2013.

VAN DER PLOEG, J. D. Trajetórias do desenvolvimento rural: pesquisa comparativa 
internacional. Sociologias, v. 27, p. 114-140, 2011.

VERNETTI JUNIOR, F. de J.; GOMES, A. da S. A.; SCHUCH, L. O. B. Sustentabilidade de sistemas de rotação e sucessão de culturas em solos de várzea no Sul do Brasil. Ciência Rural, v. 39, n. 6, p. 1708-1714, 2009.

VIZEU, F. Rural heritage of early Brazilian industrialists: its impact on managerial orientation. BAR-Brazilian Administration Review, v. 8, n. 1, p. 68-85, 2011.

Recebido em: 07 de julbo de 2015 Aceito em: 23 de abril de 2016 